\title{
On nonlinear pantograph fractional differential equations with Atangana-Baleanu-Caputo derivative
}

\author{
Mohammed S. Abdo ${ }^{1}$, Thabet Abdeljawad ${ }^{2,3,4^{*}}$ (D), Kishor D. Kucche ${ }^{5}$, Manar A. Alqudah ${ }^{6 *}$, \\ Saeed M. Ali ${ }^{7}$ and Mdi Begum Jeelani ${ }^{8}$
}

\author{
"Correspondence: \\ tabdeljawad@psu.edu.sa; \\ maalqudah@pnu.edu.sa \\ ${ }^{2}$ Department of Mathematics and \\ General Sciences, Prince Sultan \\ University, Riyadh, Saudi Arabia \\ ${ }^{6}$ Department of Mathematical \\ Sciences, Princess Nourah bint \\ Abdulrahman University, Riyadh, \\ Saudi Arabia \\ Full list of author information is \\ available at the end of the article
}

\begin{abstract}
In this paper, we obtain sufficient conditions for the existence and uniqueness results of the pantograph fractional differential equations (FDEs) with nonlocal conditions involving Atangana-Baleanu-Caputo (ABC) derivative operator with fractional orders. Our approach is based on the reduction of FDEs to fractional integral equations and on some fixed point theorems such as Banach's contraction principle and the fixed point theorem of Krasnoselskii. Further, Gronwall's inequality in the frame of the Atangana-Baleanu fractional integral operator is applied to develop adequate results for different kinds of Ulam-Hyers stabilities. Lastly, the paper includes an example to substantiate the validity of the results.
\end{abstract}

MSC: 34A08; 34D20; 97M70; 34A12

Keywords: ABC-Caputo pantograph fractional differential equation; Nonlocal conditions; Fixed point theorem; Generalized Gronwall inequality

\section{Introduction}

Fractional calculus (FC) has been growing quicker during the most recent few years, and numerous phenomena having the power-law impact have been described precisely with fractional models [1-9]. Numerous outstanding results of the fractional models have been acquired in different fields of science and engineering. One of the specificities of the FC is that we have numerous fractional derivatives (FDs) that offer the authors the chance to pick the specific FD which coincides better with a given real-world problem. The description of phenomena with memory effect is as yet a major test for the specialists. Along these lines, new tools and methods ought to be made to have the option to show a better improvement description of real-world phenomena and the existing models. In this regard, it appears that there is a need for new FDs with the nonsingular kernel. For the nonlocal FDs with the nonsingular exponential kernel, we allude to $[10,11]$, and for other local approaches of the FDs, we allude to the recent works [12, 13]. Probably the best competitor among the current kernels is the one dependent on Mittag-Leffler functions (MLF) [14]. In view of this, very lately a novel FD [14] (ABC fractional operators) was structured

(c) The Author(s) 2021. This article is licensed under a Creative Commons Attribution 4.0 International License, which permits use, sharing, adaptation, distribution and reproduction in any medium or format, as long as you give appropriate credit to the original author(s) and the source, provide a link to the Creative Commons licence, and indicate if changes were made. The images or other third party material in this article are included in the article's Creative Commons licence, unless indicated otherwise in a credit line to the material. If material is not included in the article's Creative Commons licence and your intended use is not permitted by statutory regulation or exceeds the permitted use, you will need to obtain permission directly from the copyright holder. To view a copy of this licence, visit http://creativecommons.org/licenses/by/4.0/. 
and applied to sundry real-world problems $[15,16]$. Then, in $[17,18]$, the authors deliberated the discrete versions of those new operators. For the modeling and important applications in the frame of $\mathrm{ABC}$ fractional operator, see [19-26]. Recent investigations of the existence and uniqueness of solutions for fractional differential equations (FDEs) of the impulsive, evolution, and functional problems with initial or boundary conditions can be found within the following research series [27-30] and the references therein. Recent contributions on FDEs involving ABC-FDs can be found in the articles [13,31-38].

On the other hand, the pantograph is an apparatus employed in electric trains to collect electric currents from the overload lines. This type of equation was designed by Ockendon and Tayler [39]. Pantograph equations play a pivotal role in pure and applied mathematics and physics. Motivated by their significance, a ton of scientists generalized these equations into different types and presented the solvability aspect of such problems both numerically and theoretically; for additional subtleties, see [40-46] and the references therein. Besides, some authors applied various kinds of fractional derivatives and studied the existence and stability of Ulam-Hyers, which can be found in [47-51]. However, not many works have been proposed for pantograph FDEs, especially those involving $\mathrm{ABC}$ fractional operator and nonlocal conditions.

Motivated by the above argumentations, the intent of this work is to investigate the $\mathrm{ABC}$ type pantograph FDEs with nonlocal conditions described by

$$
\begin{cases}A B C \mathbb{D}_{a^{+}}^{\vartheta} \zeta(\mathfrak{r})=f(\mathfrak{r}, \zeta(\mathfrak{r}), \varsigma(\gamma \mathfrak{r})), & \mathfrak{r} \in[a, T], 0<\vartheta \leq 1, \\ \varsigma(a)=\sum_{k=1}^{m} c_{k} \varsigma\left(\mathfrak{r}_{k}\right), & \mathfrak{r}_{k} \in(a, T),\end{cases}
$$

where $0<\gamma<1,{ }^{A B C} \mathbb{D}_{a^{+}}^{\vartheta}$ is the AB-Caputo FD of order $\vartheta, f:[a, T] \times \mathbb{R} \times \mathbb{R} \rightarrow \mathbb{R}$ is a continuous function with $f(a, \varsigma(a), \varsigma(\gamma a))=0$, and the constant $c_{k}$ satisfies the condition $\sum_{k=1}^{m} c_{k} \neq 1$. Note that, if $\gamma=1$, then our problem reduces to

$$
\begin{cases}A B C \mathbb{D}_{a^{+}}^{\vartheta} \zeta(\mathfrak{r})=f(\mathfrak{r}, \zeta(\mathfrak{r})), & \mathfrak{r} \in[a, T], 0<\vartheta \leq 1, \\ \varsigma(a)=\sum_{k=1}^{m} c_{k} \zeta\left(\mathfrak{r}_{k}\right), & \mathfrak{r}_{k} \in(a, T) .\end{cases}
$$

Therefore, if $\gamma=1$, the results acquired in the present paper are also true for $\mathrm{ABC}$-type pantograph FDEs (1.2).

Some fixed point theorems are applied to establish the existence and uniqueness of solution. The Ulam-Hyers stabilities are proved via Gronwall's inequality in the frame of $\mathrm{AB}$ fractional integral operator. The proposed problems are more generalized, also the obtained results are recent studies and an extension of the development of FDEs involving this new operator. Moreover, the analysis of the results was limited to the minimum assumptions.

This paper is formatted as follows. Section 2 provides the background materials and preliminaries required for our analysis. Section 3 is devoted to obtaining a formula of solution to the $\mathrm{ABC}$ type pantograph FDEs (1.1). In Sect. 4, we prove the existence and uniqueness of solution to problems at hand by means of some techniques of FPTs. In Sect. 5, the Ulam-Hyers and generalized Ulam-Hyers stability of the pantograph ABC-FDEs (1.1) is discussed via Gronwall's inequality in the frame of $A B$ fractional integral operator. Finally, an illustrative example is offered in Sect. 6. 


\section{Background materials and preliminaries}

Here, we recollect some requisite definitions and preliminary concepts related to our work.

Let $\mathfrak{Z}=[a, T], \mathfrak{Z}^{\prime}=(a, T) \subset \mathbb{R}$, and $\mathfrak{D}=C(\mathfrak{Z}, \mathbb{R})$ be the space of continuous functions $\varsigma: \mathfrak{Z} \rightarrow \mathbb{R}$ with the norm

$$
\|\varsigma\|=\max \{|\varsigma(\mathfrak{r})|: \mathfrak{r} \in \mathfrak{Z}\}
$$

Clearly, $\mathfrak{D}$ is a Banach space with the norm $\|\varsigma\|$.

Definition 2.1 ([14]) Let $\vartheta \in(0,1]$ and $\mathfrak{p} \in H^{1}(\mathfrak{Z})$. Then the AB-Caputo and ABRiemann-Liouville FDs of order $\vartheta$ for a function $\mathfrak{p}$ are described by

$$
A B C \mathbb{D}_{a^{+}}^{\vartheta} \mathfrak{p}(\mathfrak{r})=\frac{\mathfrak{N}(\vartheta)}{1-\vartheta} \int_{a}^{\mathfrak{r}} \mathbb{E}_{\vartheta}\left(\frac{-\vartheta}{\vartheta-1}(\mathfrak{r}-\mathfrak{s})^{\vartheta}\right) \mathfrak{p}^{\prime}(\mathfrak{s}) d \mathfrak{s}, \quad \mathfrak{r}>a,
$$

and

$$
A B R \mathbb{D}_{a^{+}}^{\vartheta} \mathfrak{p}(\mathfrak{r})=\frac{\mathfrak{N}(\vartheta)}{1-\vartheta} \frac{d}{d \mathfrak{r}} \int_{a}^{\mathfrak{r}} \mathbb{E}_{\vartheta}\left(\frac{-\vartheta}{\vartheta-1}(\mathfrak{r}-\mathfrak{s})^{\vartheta}\right) \mathfrak{p}(\mathfrak{s}) d \mathfrak{s}, \quad \mathfrak{r}>a,
$$

respectively, where $\mathfrak{N}(\vartheta)>0$ is a normalization function complying with $\mathfrak{N}(0)=\mathfrak{N}(1)=1$, and $\mathbb{E}_{\vartheta}$ is called the Mittag-Leffler function described by

$$
\mathbb{E}_{\vartheta}(\mathfrak{p})=\sum_{k=0}^{\infty} \frac{\mathfrak{p}^{k}}{\Gamma(k \vartheta+1)}, \quad \operatorname{Re}(\vartheta)>0, \mathfrak{p} \in \mathbb{C} .
$$

The associated $\mathrm{AB}$ fractional integral is specified by

$$
{ }^{A B} \mathbb{I}_{a^{+}} \mathfrak{p}(\mathfrak{r})=\frac{1-\vartheta}{\mathfrak{N}(\vartheta)} \mathfrak{p}(\mathfrak{r})+\frac{\vartheta}{\mathfrak{N}(\vartheta)} I_{a^{+}}^{\vartheta} \mathfrak{p}(\mathfrak{r}),
$$

where

$$
I_{a^{+}}^{\vartheta} \mathfrak{p}(\mathfrak{r})=\frac{1}{\Gamma(\vartheta)} \int_{a}^{\mathfrak{r}}(\mathfrak{r}-\mathfrak{s})^{\vartheta-1} \mathfrak{p}(\mathfrak{s}) d \mathfrak{s} .
$$

Lemma 2.1 ([17]) Let $\vartheta \in(0,1]$ and $\mathfrak{p} \in H^{1}(\mathfrak{Z})$, if AB-Caputo FD exists, then we have

$$
A B \mathbb{I}_{a^{+}}^{\vartheta} A B C \mathbb{D}_{a^{+}}^{\vartheta} \mathfrak{p}(\mathfrak{r})=\mathfrak{p}(\mathfrak{r})-\mathfrak{p}(a) .
$$

Definition 2.2 ([31]) The relation between the AB-Riemann-Liouville and AB-Caputo FDs is given by

$$
A B C \mathbb{D}_{a^{+}}^{\vartheta} \mathfrak{p}(\mathfrak{r})={ }^{A B R} \mathbb{D}_{a^{+}}^{\vartheta} \mathfrak{p}(\mathfrak{r})-\frac{\mathfrak{N}(\vartheta)}{1-\vartheta} \mathfrak{p}(a) \mathbb{E}_{\vartheta}\left(\frac{-\vartheta}{\vartheta-1}(\mathfrak{r}-a)^{\vartheta}\right) .
$$

Remark 2.1 Replacing $\mathfrak{p}(\mathfrak{r})$ with ${ }^{A B} \mathbb{I}_{a^{+}}^{\vartheta} \mathfrak{p}(\mathfrak{r})$ in Definition 2.2 and using Lemma 2.1, it can be shown that

$$
A B C_{\mathbb{D}_{a^{+}}^{\vartheta} A B} \mathbb{I}_{a^{+}}^{\vartheta} \mathfrak{p}(\mathfrak{r})=\mathfrak{p}(\mathfrak{r})-\mathfrak{p}(a) \mathbb{E}_{\vartheta}\left(\frac{-\vartheta}{\vartheta-1}(\mathfrak{r}-a)^{\vartheta}\right) .
$$


Hence, under the condition that $\mathfrak{p}(a)=0$, we get the identity

$$
A B C \mathbb{D}_{a^{+}}^{\vartheta} A B \mathbb{I}_{a^{+}}^{\vartheta} \mathfrak{p}(\mathfrak{r})=\mathfrak{p}(\mathfrak{r}) .
$$

Lemma $2.2([14])$ Let $\vartheta>0$. Then ${ }^{A B} \mathbb{I}_{a^{+}}$is bounded from $\mathfrak{D}$ into $\mathfrak{D}$.

Lemma $2.3([13,17])$ Let $\vartheta \in(0,1]$ and $\varpi \in \mathfrak{D}$ with $\varpi(a)=0$. Then the solution of the following problem

$$
\begin{aligned}
& { }^{A B C} D_{a^{+}}^{\vartheta} \mathfrak{p}(\mathfrak{r})=\varpi(\mathfrak{r}), \quad \mathfrak{r} \in \mathfrak{Z}, \\
& \mathfrak{p}(a)=\mathfrak{p}_{a},
\end{aligned}
$$

is given by

$$
\mathfrak{p}(\mathfrak{r})=\mathfrak{p}_{a}+\frac{1-\vartheta}{\mathfrak{N}(\vartheta)} \varpi(\mathfrak{r})+\frac{\vartheta}{\mathfrak{N}(\vartheta)} \frac{1}{\Gamma(\vartheta)} \int_{a}^{\mathfrak{r}}(\mathfrak{r}-\mathfrak{s})^{\vartheta-1} \varpi(\mathfrak{s}) d \mathfrak{s}
$$

Theorem 2.1 ([52], Banach's contraction principle) Let $\mathcal{J}$ be a Banach space, and $\mathfrak{K}$ be a nonempty closed subset of $\mathcal{J}$. If $\mathfrak{B}: \mathfrak{K} \longrightarrow \mathfrak{K}$ is a contraction, then there exists a unique fixed point of $\mathfrak{B}$.

Theorem 2.2 ([52], Krasnoselskii's fixed point theorem) Let $\mathfrak{K}$ be a nonempty, closed, convex subset of a Banach space $\mathcal{J}$. Let $\mathfrak{B}_{1}, \mathfrak{B}_{2}$ be two operators such that (i) $\mathfrak{B}_{1} u+\mathfrak{B}_{2} v \in \mathfrak{K}$, $\forall u, v \in \mathfrak{K} ;$ (ii) $\mathfrak{B}_{1}$ is compact and continuous; (iii) $\mathfrak{B}_{2}$ is a contraction mapping. Then there exists $w \in \mathfrak{K}$ such that $\mathfrak{B}_{1} w+\mathfrak{B}_{2} w=w$.

Theorem 2.3 ([13], Generalized Gronwall's inequality) Suppose that $0<\vartheta \leq 1, a(\mathfrak{r})(1-$ $\left.\frac{1-\vartheta}{\mathfrak{N}(\vartheta)} b(\mathfrak{r})\right)^{-1}$ is a nonnegative, nondecreasing, and locally integrable function on $[c, d)$, $\frac{\vartheta b(t)}{\mathfrak{N}(\vartheta)}\left(1-\frac{1-\vartheta}{\mathfrak{N}(\vartheta)} b(\mathfrak{r})\right)^{-1}$ is nonnegative and bounded on $[c, d)$, and $\sigma(\mathfrak{r})$ is nonnegative and locally integrable on $[c, d)$ with

$$
\sigma(\mathfrak{r}) \leq a(\mathfrak{r})+b(\mathfrak{r})\left({ }^{A B} \mathbb{I}_{a^{+}}^{\vartheta} \sigma\right)(\mathfrak{r})
$$

Then

$$
\sigma(\mathfrak{r}) \leq \frac{a(\mathfrak{r}) \mathfrak{N}(\vartheta)}{\mathfrak{N}(\vartheta)-(1-\vartheta) b(\mathfrak{r})} \mathbb{E}_{\vartheta}\left(\frac{\vartheta b(\mathfrak{r})(\mathfrak{r}-a)^{\vartheta}}{\mathfrak{N}(\vartheta)-(1-\vartheta) b(\mathfrak{r})}\right) .
$$

\section{Formulas of solution}

This section is devoted to obtaining formulas of solution to linear problems corresponding to (1.1).

Theorem 3.1 Let $0<\vartheta \leq 1, \sum_{k=1}^{m} c_{k} \neq 1$, and let $\varpi \in \mathfrak{D}$ with $\varpi(a)=0$. A function $\varsigma \in \mathfrak{D}$ is a solution of the fractional integral equation (FIE)

$$
\begin{aligned}
\zeta(\mathfrak{r})= & \frac{1}{1-\sum_{k=1}^{m} c_{k}}\left(\frac{1-\vartheta}{\mathfrak{N}(\vartheta)} \sum_{k=1}^{m} c_{k} \varpi\left(\mathfrak{r}_{k}\right)+\frac{\vartheta}{\mathfrak{N}(\vartheta)} \sum_{k=1}^{m} c_{k} I_{a^{+}}^{\vartheta} \varpi\left(\mathfrak{r}_{k}\right)\right) \\
& +\frac{1-\vartheta}{\mathfrak{N}(\vartheta)} \varpi(\mathfrak{r})+\frac{\vartheta}{\mathfrak{N}(\vartheta)} I_{a^{+}}^{\vartheta}(\mathfrak{r})
\end{aligned}
$$


if and only if $\varsigma$ is a solution of the ABC-problem

$$
\begin{aligned}
& { }^{A B C} \mathbb{D}_{a^{+}}^{\vartheta} \zeta(\mathfrak{r})=\varpi(\mathfrak{r}), \quad \mathfrak{r} \in \mathfrak{Z}, \\
& \zeta(a)=\sum_{k=1}^{m} c_{k} \zeta\left(\mathfrak{r}_{k}\right), \quad \mathfrak{r}_{k} \in \mathfrak{Z}^{\prime} .
\end{aligned}
$$

Proof Assume that $\varsigma$ satisfies the first equation of (3.2). From Lemma 2.3, we have

$$
\zeta(\mathfrak{r})=\varsigma(a)+\frac{1-\vartheta}{\mathfrak{N}(\vartheta)} \varpi(\mathfrak{r})+\frac{\vartheta}{\mathfrak{N}(\vartheta)} \frac{1}{\Gamma(\vartheta)} \int_{a}^{\mathfrak{r}}(\mathfrak{r}-\mathfrak{s})^{\vartheta-1} \varpi(\mathfrak{s}) d \mathfrak{s} .
$$

Now, if we replace $\mathfrak{r}=\mathfrak{r}_{k}$ and multiply both sides by $c_{k}$ in (3.3), we get

$$
c_{k} \zeta\left(\mathfrak{r}_{k}\right)=c_{k} \zeta(a)+\frac{1-\vartheta}{\mathfrak{N}(\vartheta)} c_{k} \varpi\left(\mathfrak{r}_{k}\right)+\frac{\vartheta c_{k}}{\mathfrak{N}(\vartheta)} \frac{1}{\Gamma(\vartheta)} \int_{a}^{\mathfrak{r}_{k}}\left(\mathfrak{r}_{k}-\mathfrak{s}\right)^{\vartheta-1} \varpi(\mathfrak{s}) d \mathfrak{s} .
$$

From the nonlocal condition, we get

$$
\begin{aligned}
\varsigma(a) & =\sum_{k=1}^{m} c_{k} \zeta\left(\mathfrak{r}_{k}\right) \\
& =\sum_{k=1}^{m} c_{k} \varsigma(a)+\frac{1-\vartheta}{\mathfrak{N}(\vartheta)} \sum_{k=1}^{m} c_{k} \varpi\left(\mathfrak{r}_{k}\right)+\frac{\vartheta}{\mathfrak{N}(\vartheta)} \sum_{k=1}^{m} c_{k} I_{a^{+}}^{\vartheta} \varpi\left(\mathfrak{r}_{k}\right),
\end{aligned}
$$

which implies

$$
\zeta(a)=\frac{1}{1-\sum_{k=1}^{m} c_{k}}\left(\frac{1-\vartheta}{\mathfrak{N}(\vartheta)} \sum_{k=1}^{m} c_{k} \varpi\left(\mathfrak{r}_{k}\right)+\frac{\vartheta}{\mathfrak{N}(\vartheta)} \sum_{k=1}^{m} c_{k} I_{a^{+}}^{\vartheta} \varpi\left(\mathfrak{r}_{k}\right)\right) .
$$

By matching the two equations (3.3) and (3.5), we get

$$
\begin{aligned}
\zeta(\mathfrak{r})= & \frac{1}{1-\sum_{k=1}^{m} c_{k}}\left(\frac{1-\vartheta}{\mathfrak{N}(\vartheta)} \sum_{k=1}^{m} c_{k} \varpi\left(\mathfrak{r}_{k}\right)+\frac{\vartheta}{\mathfrak{N}(\vartheta)} \sum_{k=1}^{m} c_{k} I_{a^{+}}^{\vartheta} \varpi\left(\mathfrak{r}_{k}\right)\right) \\
& +\frac{1-\vartheta}{\mathfrak{N}(\vartheta)} \varpi(\mathfrak{r})+\frac{\vartheta}{\mathfrak{N}(\vartheta)} I_{a^{+}}^{\vartheta} \varpi(\mathfrak{r}) .
\end{aligned}
$$

Thus (3.1) is satisfied.

Conversely, suppose that $\zeta$ satisfies equation (3.1). Applying ${ }^{A B C} \mathbb{D}_{a^{+}}^{\vartheta}$ on both sides of (3.1), then using Remark 2.1, and from the fact ${ }^{A B C} \mathbb{D}_{a^{+}}^{\vartheta}(k)=0$, for $k=$ constant, we find that

$$
\begin{aligned}
A B C & \mathbb{D}_{a^{+}}^{\vartheta} \zeta(\mathfrak{r})= \\
& A B C \mathbb{D}_{a^{+}}^{\vartheta}\left[\frac{1}{1-\sum_{k=1}^{m} c_{k}}\left(\frac{1-\vartheta}{\mathfrak{N}(\vartheta)} \sum_{k=1}^{m} c_{k} \varpi\left(\mathfrak{r}_{k}\right)+\frac{\vartheta}{\mathfrak{N}(\vartheta)} \sum_{k=1}^{m} c_{k} I_{a^{+}}^{\vartheta} \varpi\left(\mathfrak{r}_{k}\right)\right)\right] \\
& +{ }^{A B C} \mathbb{D}_{a^{+}}^{\vartheta}\left[\frac{1-\vartheta}{\mathfrak{N}(\vartheta)} \varpi(\mathfrak{r})+\frac{\vartheta}{\mathfrak{N}(\vartheta)} I_{a^{+}}^{\vartheta} \varpi(\mathfrak{r})\right] \\
= & A B C \mathbb{D}_{a^{+}}^{\vartheta} \mathbb{I}_{a^{+}}^{\vartheta} \varpi(\mathfrak{r}) \\
= & \varpi(\mathfrak{r}) .
\end{aligned}
$$


On the other hand, by taking $\mathfrak{r} \rightarrow a$ on both sides of (3.1), then using the fact that $\varpi(a)=$ 0 and $\lim _{\mathfrak{r} \rightarrow a} I_{a^{+}}^{\vartheta} \varpi(\mathfrak{r})=I_{a^{+}}^{\vartheta} \varpi(a)=0$, we get

$$
\begin{aligned}
\varsigma(a)= & \frac{1}{1-\sum_{k=1}^{m} c_{k}}\left(\frac{1-\vartheta}{\mathfrak{N}(\vartheta)} \sum_{k=1}^{m} c_{k} \varpi\left(\mathfrak{r}_{k}\right)+\frac{\vartheta}{\mathfrak{N}(\vartheta)} \sum_{k=1}^{m} c_{k} I_{a^{+}}^{\vartheta} \varpi\left(\mathfrak{r}_{k}\right)\right) \\
& +\frac{1-\vartheta}{\mathfrak{N}(\vartheta)} \varpi(a)+\frac{\vartheta}{\mathfrak{N}(\vartheta)} I_{a^{+}}^{\vartheta} \varpi(a) \\
= & \frac{1}{1-\sum_{k=1}^{m} c_{k}}\left(\frac{1-\vartheta}{\mathfrak{N}(\vartheta)} \sum_{k=1}^{m} c_{k} \varpi\left(\mathfrak{r}_{k}\right)+\frac{\vartheta}{\mathfrak{N}(\vartheta)} \sum_{k=1}^{m} c_{k} I_{a^{+}} \varpi\left(\mathfrak{r}_{k}\right)\right) .
\end{aligned}
$$

Substitute $\mathfrak{r}=\mathfrak{r}_{k}$ and multiply by $c_{k}$ in (3.1). Then we derive

$$
\begin{aligned}
\sum_{k=1}^{m} c_{k} \zeta\left(\mathfrak{r}_{k}\right)= & \left(\frac{\sum_{k=1}^{m} c_{k}}{1-\sum_{k=1}^{m} c_{k}}\left(\frac{1-\vartheta}{\mathfrak{N}(\vartheta)} \sum_{k=1}^{m} c_{k} \varpi\left(\mathfrak{r}_{k}\right)+\frac{\vartheta}{\mathfrak{N}(\vartheta)} \sum_{k=1}^{m} c_{k} I_{a^{+}}^{\vartheta} \varpi\left(\mathfrak{r}_{k}\right)\right)\right) \\
& +\frac{1-\vartheta}{\mathfrak{N}(\vartheta)} \sum_{k=1}^{m} c_{k} \varpi\left(\mathfrak{r}_{k}\right)+\frac{\vartheta}{\mathfrak{N}(\vartheta)} \sum_{k=1}^{m} c_{k} I_{a^{+}} c_{k} \varpi\left(\mathfrak{r}_{k}\right) \\
= & \left(\frac{\sum_{k=1}^{m} c_{k}}{1-\sum_{k=1}^{m} c_{k}}+1\right)\left(\frac{1-\vartheta}{\mathfrak{N}(\vartheta)} \sum_{k=1}^{m} c_{k} \varpi\left(\mathfrak{r}_{k}\right)+\frac{\vartheta}{\mathfrak{N}(\vartheta)} \sum_{k=1}^{m} c_{k} I_{a^{+}} \varpi\left(\mathfrak{r}_{k}\right)\right) \\
= & \frac{1}{1-\sum_{k=1}^{m} c_{k}}\left(\frac{1-\vartheta}{\mathfrak{N}(\vartheta)} \sum_{k=1}^{m} c_{k} \varpi\left(\mathfrak{r}_{k}\right)+\frac{\vartheta}{\mathfrak{N}(\vartheta)} \sum_{k=1}^{m} c_{k} I_{a^{+}} \varpi\left(\mathfrak{r}_{k}\right)\right) .
\end{aligned}
$$

It follows from (3.6) and (3.7) that

$$
\zeta(a)=\sum_{k=1}^{m} c_{k} \varsigma\left(\mathfrak{r}_{k}\right)
$$

As a result of Theorem 3.1, we have the subsequent theorem.

Theorem 3.2 Let $0<\vartheta \leq 1, \sum_{k=1}^{m} c_{k} \neq 1$, and $f: \mathfrak{Z} \times \mathbb{R} \times \mathbb{R} \rightarrow \mathbb{R}$ be a continuous function along with $f(a, \varsigma(a), \varsigma(\gamma a))=0, \varsigma \in \mathfrak{D}$. Then the ABC-type pantograph FDEs (1.1) are equivalent to the following FIE:

$$
\begin{aligned}
\varsigma(\mathfrak{r})= & \frac{1}{1-\sum_{k=1}^{m} c_{k}}\left(\frac{1-\vartheta}{\mathfrak{N}(\vartheta)} \sum_{k=1}^{m} c_{k} f\left(\mathfrak{r}_{k}, \varsigma\left(\mathfrak{r}_{k}\right), \varsigma\left(\gamma\left(\mathfrak{r}_{k}\right)\right)\right)\right. \\
& \left.+\frac{\vartheta}{\mathfrak{N}(\vartheta)} \sum_{k=1}^{m} c_{k} I_{a^{+}}^{\vartheta} f\left(\mathfrak{r}_{k}, \varsigma\left(\mathfrak{r}_{k}\right), \varsigma\left(\gamma\left(\mathfrak{r}_{k}\right)\right)\right)\right) \\
& +\frac{1-\vartheta}{\mathfrak{N}(\vartheta)} f(\mathfrak{r}, \varsigma(\mathfrak{r}), \varsigma(\gamma \mathfrak{r}))+\frac{\vartheta}{\mathfrak{N}(\vartheta)} I_{a^{+}}^{\vartheta} f(\mathfrak{r}, \varsigma(\mathfrak{r}), \varsigma(\gamma \mathfrak{r})), \quad \mathfrak{r} \in \mathfrak{Z} .
\end{aligned}
$$

\section{Existence and uniqueness theorems}

This section is devoted to proving the existence and uniqueness theorems for the $\mathrm{ABC}$ type pantograph FDEs (1.1). Before proceeding with the main findings, we are obligated to provide the following assumption: 
$\left(A_{1}\right)$ The function $f: \mathfrak{Z} \times \mathbb{R} \times \mathbb{R} \rightarrow \mathbb{R}$ is continuous, and there exists $L_{f}>0$ such that

$$
|f(\mathfrak{r}, u, \bar{u})-f(\mathfrak{r}, v, \bar{v})| \leq L_{f}(|u-v|+|\bar{u}-\bar{v}|), \quad \mathfrak{r} \in \mathfrak{Z} \text {, and } u, v, \bar{u}, \bar{v} \in \mathbb{R} .
$$

Theorem 4.1 Suppose that hypothesis $\left(A_{1}\right)$ holds. Then the ABC-type pantograph FDEs (1.1) have a unique solution, provided that

$$
\begin{aligned}
\mathfrak{P}_{1} & :=\left(\frac{(1-\vartheta)}{1-\sum_{k=1}^{m} c_{k}}+\frac{1}{\Gamma(\vartheta)}\left[\frac{1}{\left(1-\sum_{k=1}^{m} c_{k}\right)} \sum_{k=1}^{m} c_{k}\left(\mathfrak{r}_{k}-a\right)^{\vartheta}+(T-a)^{\vartheta}\right]\right) \frac{2 L_{f}}{\mathfrak{N}(\vartheta)} \\
& <1 .
\end{aligned}
$$

Proof Define the operator $\mathbb{T}: \mathfrak{D} \rightarrow \mathfrak{D}$ by $\mathbb{T} \varsigma=\varsigma, \varsigma \in \mathfrak{D}$, i.e.,

$$
\begin{aligned}
(\mathbb{T} \varsigma)(\mathfrak{r})= & \frac{1}{1-\sum_{k=1}^{m} c_{k}}\left(\frac{1-\vartheta}{\mathfrak{N}(\vartheta)} \sum_{k=1}^{m} c_{k} f\left(\mathfrak{r}_{k}, \varsigma\left(\mathfrak{r}_{k}\right), \varsigma\left(\gamma\left(\mathfrak{r}_{k}\right)\right)\right)\right. \\
& \left.+\frac{\vartheta}{\mathfrak{N}(\vartheta)} \sum_{k=1}^{m} c_{k} I_{a^{+}}^{\vartheta} f\left(\mathfrak{r}_{k}, \varsigma\left(\mathfrak{r}_{k}\right), \varsigma\left(\gamma\left(\mathfrak{r}_{k}\right)\right)\right)\right) \\
& +\frac{1-\vartheta}{\mathfrak{N}(\vartheta)} f(\mathfrak{r}, \varsigma(\mathfrak{r}), \varsigma(\gamma \mathfrak{r}))+\frac{\vartheta}{\mathfrak{N}(\vartheta)} I_{a}^{\vartheta} f(\mathfrak{r}, \varsigma(\mathfrak{r}), \varsigma(\gamma \mathfrak{r})) .
\end{aligned}
$$

The operator $\mathbb{T}$ is well defined, that is, $\mathbb{T}(\mathfrak{D}) \subseteq \mathfrak{D}$. Indeed, for any $\varsigma \in \mathfrak{D}, f(\cdot, \varsigma(\cdot), \varsigma(\gamma(\cdot)))$ is continuous. Besides, by Lemma 2.2, $\mathbb{T} \varsigma \in \mathfrak{D}$. Also, by Lemma 2.1 with Remark 2.1, we end up at

$$
\begin{aligned}
{ }^{A B C} \mathbb{D}_{a^{+}}^{\vartheta}(\mathbb{T} \zeta)(\mathfrak{r}) & ={ }^{A B C} \mathbb{D}_{a^{+}}^{\vartheta} \zeta(a)+{ }^{A B C} \mathbb{D}_{a^{+}}{ }^{A B} \mathbb{I}_{a^{+}}^{\vartheta} f(\mathfrak{r}, \varsigma(\mathfrak{r}), \varsigma(\gamma \mathfrak{r})) \\
& =f(\mathfrak{r}, \varsigma(\mathfrak{r}), \varsigma(\gamma \mathfrak{r})) .
\end{aligned}
$$

Since $f(\mathfrak{r}, \cdot, \cdot)$ is continuous on $[a, T]$, then ${ }^{A B C} \mathbb{D}_{a^{+}}^{\vartheta}(\mathbb{T} \zeta)(\mathfrak{r}) \in \mathfrak{D}$.

Now, we need to prove that $\mathbb{T}$ is a condensing map. Let $\zeta, \bar{\zeta} \in \mathfrak{D}$ and $\mathfrak{r} \in \mathfrak{Z}$. Then

$$
\begin{aligned}
|(\mathbb{T} \zeta)(\mathfrak{r})-(\mathbb{T} \bar{\zeta})(\mathfrak{r})| & \\
\leq & \frac{1}{1-\sum_{k=1}^{m} c_{k}}\left(\frac{1-\vartheta}{\mathfrak{N}(\vartheta)} \sum_{k=1}^{m} c_{k}\left|f\left(\mathfrak{r}_{k}, \zeta\left(\mathfrak{r}_{k}\right), \varsigma\left(\gamma \mathfrak{r}_{k}\right)\right)-f\left(\mathfrak{r}_{k}, \bar{\zeta}\left(\mathfrak{r}_{k}\right), \bar{\zeta}\left(\gamma \mathfrak{r}_{k}\right)\right)\right|\right. \\
& \left.+\frac{\vartheta}{\mathfrak{N}(\vartheta)} \sum_{k=1}^{m} c_{k} I_{a^{+}}^{\vartheta}\left|f\left(\mathfrak{r}_{k}, \zeta\left(\mathfrak{r}_{k}\right), \zeta\left(\gamma \mathfrak{r}_{k}\right)\right)-f\left(\mathfrak{r}_{k}, \bar{\zeta}\left(\mathfrak{r}_{k}\right), \bar{\zeta}\left(\gamma \mathfrak{r}_{k}\right)\right)\right|\right) \\
& +\frac{1-\vartheta}{\mathfrak{N}(\vartheta)} \mid f(\mathfrak{r}, \zeta(\mathfrak{r}), \zeta(\gamma \mathfrak{r}))-f(\mathfrak{r}, \bar{\zeta}(\mathfrak{r}), \bar{\zeta}(\gamma \mathfrak{r}) \mid \\
& +\frac{\vartheta}{\mathfrak{N}(\vartheta)} I_{a^{+}}^{\vartheta}|f(\mathfrak{r}, \zeta(\mathfrak{r}), \varsigma(\gamma \mathfrak{r}))-f(\mathfrak{r}, \bar{\zeta}(\mathfrak{r}), \bar{\zeta}(\gamma \mathfrak{r}))| .
\end{aligned}
$$

By assumption $\left(A_{1}\right)$, we obtain

$$
\begin{aligned}
& I_{a^{+}}^{\vartheta}\left|f\left(\mathfrak{r}_{k}, \varsigma\left(\mathfrak{r}_{k}\right), \varsigma\left(\gamma\left(\mathfrak{r}_{k}\right)\right)\right)-f\left(\mathfrak{r}_{k}, \bar{\zeta}\left(\mathfrak{r}_{k}\right), \bar{\zeta}\left(\gamma\left(\mathfrak{r}_{k}\right)\right)\right)\right| \\
& \quad \leq \frac{1}{\Gamma(\vartheta)} \int_{a}^{\mathfrak{r}_{k}}\left(\mathfrak{r}_{k}-\mathfrak{s}\right)^{\vartheta-1}|f(\mathfrak{s}, \varsigma(\mathfrak{s}), \varsigma(\gamma(\mathfrak{s})))-f(\mathfrak{s}, \bar{\zeta}(\mathfrak{s}), \bar{\zeta}(\gamma(\mathfrak{s})))| d \mathfrak{s}
\end{aligned}
$$




$$
\begin{aligned}
& =\frac{1}{\Gamma(\vartheta)} \int_{a}^{\mathfrak{r}_{k}}\left(\mathfrak{r}_{k}-\mathfrak{s}\right)^{\vartheta-1} L_{f}(|\varsigma(\mathfrak{s})-\bar{\varsigma}(\mathfrak{s})|+|\varsigma(\gamma(\mathfrak{s}))-\bar{\varsigma}(\gamma(\mathfrak{s}))|) d \mathfrak{s} \\
\leq & \frac{2 L_{f}\left(\mathfrak{r}_{k}-a\right)^{\vartheta}}{\Gamma(\vartheta+1)}\|\varsigma-\bar{\varsigma}\| .
\end{aligned}
$$

Similarly,

$$
\begin{aligned}
& I_{a^{+}}^{\vartheta}|f(\mathfrak{r}, \varsigma(\mathfrak{r}), \varsigma(\gamma \mathfrak{r}))-f(\mathfrak{r}, \bar{\zeta}(\mathfrak{r}), \bar{\zeta}(\gamma \mathfrak{r}))| \\
& \quad \leq \frac{2 L_{f}(T-a)^{\vartheta}}{\Gamma(\vartheta+1)}\|\varsigma-\bar{\varsigma}\| .
\end{aligned}
$$

Therefore,

$$
\begin{aligned}
\|( & \mathbb{T} \varsigma)-(\mathbb{T} \bar{\zeta}) \| \\
= & \max _{\mathfrak{r} \in \mathfrak{J}}|(\mathbb{T} \varsigma)(\mathfrak{r})-(\mathbb{T} \bar{\zeta})(\mathfrak{r})| \\
\leq & \frac{1}{1-\sum_{k=1}^{m} c_{k}}\left(\frac{2 L_{f}(1-\vartheta)}{\mathfrak{N}(\vartheta)} \sum_{k=1}^{m} c_{k}+\frac{2 L_{f}}{\mathfrak{N}(\vartheta) \Gamma(\vartheta)} \sum_{k=1}^{m} c_{k}\left(\mathfrak{r}_{k}-a\right)^{\vartheta}\right)\|\varsigma-\bar{\zeta}\| \\
& +\left(\frac{2 L_{f}(1-\vartheta)}{\mathfrak{N}(\vartheta)}+\frac{2 L_{f}(T-a)^{\vartheta}}{\mathfrak{N}(\vartheta) \Gamma(\vartheta)}\right)\|\varsigma-\bar{\zeta}\| \\
= & \left.\frac{4(1-\vartheta) \sum_{k=1}^{m} c_{k}}{1-\sum_{k=1}^{m} c_{k}}+\frac{2}{\Gamma(\vartheta)}\left[\frac{1}{1-\sum_{k=1}^{m} c_{k}} \sum_{k=1}^{m} c_{k}\left(\mathfrak{r}_{k}-a\right)^{\vartheta}+(T-a)^{\vartheta}\right]\right) \\
& \quad \times \frac{L_{f}}{\mathfrak{N}(\vartheta)}\|\varsigma-\bar{\varsigma}\| \\
= & \mathfrak{P}_{1}\|\varsigma-\bar{\zeta}\| .
\end{aligned}
$$

Condition (4.1) shows that $\mathbb{T}$ is a condensing operator. Hence, by Theorem 2.1, $\mathbb{T}$ has a unique fixed point.

Theorem 4.2 Suppose that hypothesis $\left(A_{1}\right)$ holds. Then there exists at least one solution of the ABC-type pantograph FDEs (1.1), provided that condition (4.1) is satisfied.

Proof Consider the operator $\mathbb{T}: \mathfrak{D} \rightarrow \mathfrak{D}$ defined by

$$
(\mathbb{T} \varsigma)(\mathfrak{r})=\left(\mathbb{T}_{1} \varsigma\right)(\mathfrak{r})+\left(\mathbb{T}_{2} \zeta\right)(\mathfrak{r}), \quad \varsigma \in \mathfrak{D}, \mathfrak{r} \in \mathfrak{Z},
$$

where

$$
\begin{aligned}
\left(\mathbb{T}_{1} \varsigma\right)(\mathfrak{r})= & \frac{1}{1-\sum_{k=1}^{m} c_{k}}\left(\frac{1-\vartheta}{\mathfrak{N}(\vartheta)} \sum_{k=1}^{m} c_{k} f\left(\mathfrak{r}_{k}, \zeta\left(\mathfrak{r}_{k}\right), \varsigma\left(\gamma \mathfrak{r}_{k}\right)\right)\right. \\
& \left.+\frac{\vartheta}{\mathfrak{N}(\vartheta)} \sum_{k=1}^{m} c_{k} I_{a^{+}}^{\vartheta} f\left(\mathfrak{r}_{k}, \zeta\left(\mathfrak{r}_{k}\right), \zeta\left(\gamma \mathfrak{r}_{k}\right)\right)\right)
\end{aligned}
$$

and

$$
\left(\mathbb{T}_{2} \varsigma\right)(\mathfrak{r})=\frac{1-\vartheta}{\mathfrak{N}(\vartheta)} f(\mathfrak{r}, \varsigma(\mathfrak{r}), \varsigma(\gamma \mathfrak{r}))+\frac{\vartheta}{\mathfrak{N}(\vartheta)} I_{a^{+}}^{\vartheta} f(\mathfrak{r}, \varsigma(\mathfrak{r}), \varsigma(\gamma \mathfrak{r})) .
$$


Since $f: \mathfrak{Z} \times \mathbb{R} \times \mathbb{R} \rightarrow \mathbb{R}$ is continuous, $\mu_{f}:=\max \{|f(\mathfrak{r}, 0,0)|: \mathfrak{r} \in \mathfrak{Z}\}$ exists. Let

$$
B_{\xi}=\{\varsigma \in \mathfrak{D}:\|\varsigma\| \leq \xi\}
$$

with the radius

$$
\xi \geq \frac{\mathfrak{P}_{2}}{1-\mathfrak{P}_{1}}
$$

where

$$
\mathfrak{P}_{2}:=\left(\frac{1-\vartheta}{1-\sum_{k=1}^{m} c_{k}}+\frac{1}{\Gamma(\vartheta)}\left[\frac{1}{\left(1-\sum_{k=1}^{m} c_{k}\right)} \sum_{k=1}^{m} c_{k}\left(\mathfrak{r}_{k}-a\right)^{\vartheta}+(T-a)^{\vartheta}\right]\right) \frac{\mu_{f}}{\mathfrak{N}(\vartheta)}
$$

We will complete the proof in the following several steps.

Step1: We show that $\mathbb{T}_{1} \varsigma+\mathbb{T}_{2} v \in B_{\xi}$ for all $\varsigma, v \in B_{\xi}$.

By (4.2), we have

$$
\begin{aligned}
\left|\left(\mathbb{T}_{1} \varsigma\right)(\mathfrak{r})\right| \leq & \frac{1}{1-\sum_{k=1}^{m} c_{k}}\left(\frac{1-\vartheta}{\mathfrak{N}(\vartheta)} \sum_{k=1}^{m} c_{k}\left|f\left(\mathfrak{r}_{k}, \varsigma\left(\mathfrak{r}_{k}\right), \varsigma\left(\gamma \mathfrak{r}_{k}\right)\right)\right|\right. \\
& \left.+\frac{\vartheta}{\mathfrak{N}(\vartheta)} \sum_{k=1}^{m} c_{k} I_{a^{+}}^{\vartheta}\left|f\left(\mathfrak{r}_{k}, \varsigma\left(\mathfrak{r}_{k}\right), \varsigma\left(\gamma \mathfrak{r}_{k}\right)\right)\right|\right) .
\end{aligned}
$$

Using hypothesis $\left(A_{1}\right)$, for $\varsigma \in B_{\xi}$ and for any $\mathfrak{r} \in \mathfrak{Z}$, we have

$$
\begin{aligned}
|f(\mathfrak{r}, \varsigma(\mathfrak{r}), \varsigma(\gamma \mathfrak{r}))| & \leq|f(\mathfrak{r}, \varsigma(\mathfrak{r}), \varsigma(\gamma \mathfrak{r}))-f(\mathfrak{r}, 0,0)|+|f(\mathfrak{r}, 0,0)| \\
& \leq L_{f}|\varsigma(\mathfrak{r})|+L_{f}|\varsigma(\gamma \mathfrak{r})|+\mu_{f} \\
& \leq 2 L_{f} \xi+\mu_{f} .
\end{aligned}
$$

Further, by using (4.8), for any $\mathfrak{r} \in \mathfrak{Z}$, we have

$$
\begin{aligned}
I_{a^{+}}^{\vartheta}|f(\mathfrak{r}, \varsigma(\mathfrak{r}), \varsigma(\gamma \mathfrak{r}))| & \leq I_{a^{+}}^{\vartheta}\left(2 L_{f} \xi+\mu_{f}\right) \\
& =\left(2 L_{f} \xi+\mu_{f}\right) \frac{(\mathfrak{r}-a)^{\vartheta}}{\Gamma(\vartheta+1)} .
\end{aligned}
$$

Using inequalities (4.8) and (4.9) into inequality (4.7), we obtain

$$
\begin{aligned}
\left\|\left(\mathbb{T}_{1} \varsigma\right)\right\|= & \max _{\mathfrak{r} \in \mathfrak{Z}}\left|\left(\mathbb{T}_{1} \varsigma\right)(\mathfrak{r})\right| \\
\leq & \frac{\left(2 L_{f} \xi+\mu_{f}\right)}{\mathfrak{N}(\vartheta)\left(1-\sum_{k=1}^{m} c_{k}\right)}\left((1-\vartheta) \sum_{k=1}^{m} c_{k}+\frac{1}{\Gamma(\vartheta)} \sum_{k=1}^{m} c_{k}\left(\mathfrak{r}_{k}-a\right)^{\vartheta}\right) \\
= & \left((1-\vartheta) \frac{\sum_{k=1}^{m} c_{k}}{1-\sum_{k=1}^{m} c_{k}}+\frac{1}{\Gamma(\vartheta)\left(1-\sum_{k=1}^{m} c_{k}\right)} \sum_{k=1}^{m} c_{k}\left(\mathfrak{r}_{k}-a\right)^{\vartheta}\right) \frac{2 L_{f}}{\mathfrak{N}(\vartheta)} \xi \\
& +\left((1-\vartheta) \frac{1}{1-\sum_{k=1}^{m} c_{k} c_{k}}+\frac{1}{\Gamma(\vartheta)\left(1-\sum_{k=1}^{m} c_{k}\right)} \sum_{k=1}^{m} c_{k}\left(\mathfrak{r}_{k}-a\right)^{\vartheta}\right) \frac{\mu_{f}}{\mathfrak{N}(\vartheta)}
\end{aligned}
$$


Also, for $v \in B_{\xi}$,

$$
\begin{aligned}
\left\|\left(\mathbb{T}_{2} v\right)\right\|= & \max _{\mathfrak{r} \in \mathfrak{J}}\left|\left(\mathbb{T}_{2} v\right)(\mathfrak{r})\right| \\
\leq & \max _{\mathfrak{r} \in \mathfrak{J}}\left(\frac{1-\vartheta}{\mathfrak{N}(\vartheta)}|f(\mathfrak{r}, v(\mathfrak{r}), v(\gamma \mathfrak{r}))|+\frac{\vartheta}{\mathfrak{N}(\vartheta)} I_{a^{+}}^{\vartheta}|f(\mathfrak{r}, v(\mathfrak{r}), v(\gamma \mathfrak{r}))|\right) \\
\leq & \frac{(1-\vartheta)}{\mathfrak{N}(\vartheta)}\left(2 L_{f} \xi+\mu_{f}\right)+\frac{\left(2 L_{f} \xi+\mu_{f}\right)}{\mathfrak{N}(\vartheta)} \frac{(T-a)^{\vartheta}}{\Gamma(\vartheta)} \\
= & \left((1-\vartheta)+\frac{(T-a)^{\vartheta}}{\Gamma(\vartheta)}\right) \frac{2 L_{f}}{\mathfrak{N}(\vartheta)} \xi \\
& +\left((1-\vartheta)+\frac{(T-a)^{\vartheta}}{\Gamma(\vartheta)}\right) \frac{\mu_{f}}{\mathfrak{N}(\vartheta)} .
\end{aligned}
$$

Inequalities (4.10) and (4.11) give

$$
\begin{aligned}
&\left\|\left(\mathbb{T}_{1} \varsigma\right)+\left(\mathbb{T}_{2} v\right)\right\| \\
& \leq\left\|\left(\mathbb{T}_{1} \varsigma\right)\right\|+\left\|\left(\mathbb{T}_{2} v\right)\right\| \\
& \leq\left(\frac{1-\vartheta}{1-\sum_{k=1}^{m} c_{k}}+\frac{1}{\Gamma(\vartheta)}\left[\frac{1}{\left(1-\sum_{k=1}^{m} c_{k}\right)} \sum_{k=1}^{m} c_{k}\left(\mathfrak{r}_{k}-a\right)^{\vartheta}+(T-a)^{\vartheta}\right]\right) \frac{2 L_{f}}{\mathfrak{N}(\vartheta)} \xi \\
&+\left(\frac{1-\vartheta}{1-\sum_{k=1}^{m} c_{k}}+\frac{1}{\Gamma(\vartheta)}\left[\frac{1}{\left(1-\sum_{k=1}^{m} c_{k}\right)} \sum_{k=1}^{m} c_{k}\left(\mathfrak{r}_{k}-a\right)^{\vartheta}+(T-a)^{\vartheta}\right]\right) \frac{\mu_{f}}{\mathfrak{N}(\vartheta)} \\
&= \mathfrak{P}_{1} \xi+\mathfrak{P}_{2} .
\end{aligned}
$$

Using (4.1) and (4.5), we get

$\left\|\mathbb{T}_{1} \varsigma+\mathbb{T}_{2} v\right\| \leq \xi$

Thus, $\mathbb{T}_{1} \varsigma+\mathbb{T}_{2} v \in B_{\xi}$ for all $\zeta, v \in B_{\xi}$.

Step 2. $\mathbb{T}_{1}$ is a condensing map. This is evident due to $\mathbb{T}$ is a contraction map.

Step 3: $\mathbb{T}_{2}$ is continuous and compact.

$\mathbb{T}_{2}: B_{\xi} \rightarrow B_{\xi}$ is continuous due to $f$ is continuous. Indeed, let $\varsigma_{n}$ be a sequence such that $\varsigma_{n} \rightarrow \varsigma$ in $\mathfrak{D}$. Then, for all $\mathfrak{r} \in \mathfrak{Z}$, one has

$$
\begin{aligned}
\left|\left(\mathbb{T}_{2} \varsigma_{n}(\mathfrak{r})\right)-\left(\mathbb{T}_{2} \varsigma(\mathfrak{r})\right)\right| \leq & \frac{1-\vartheta}{\mathfrak{N}(\vartheta)}\left|f\left(\mathfrak{r}, \varsigma_{n}(\mathfrak{r}), \varsigma_{n}(\gamma \mathfrak{r})\right)-f(\mathfrak{r}, \varsigma(\mathfrak{r}), \varsigma(\gamma \mathfrak{r}))\right| \\
& +\frac{\vartheta}{\mathfrak{N}(\vartheta)} I_{a^{+}}^{\vartheta}\left|f\left(\mathfrak{r}, \varsigma_{n}(\mathfrak{r}), \varsigma_{n}(\gamma \mathfrak{r})\right)-f(\mathfrak{r}, \varsigma(\mathfrak{r}), \varsigma(\gamma \mathfrak{r}))\right| \\
\leq & \frac{1-\vartheta}{\mathfrak{N}(\vartheta)}\left\|f\left(\cdot, \varsigma_{n}(\cdot), \varsigma_{n}(\gamma(\cdot))\right)-f(\cdot, \varsigma(\cdot), \varsigma(\gamma(\cdot)))\right\| \\
& +\frac{(T-a)^{\vartheta}}{\mathfrak{N}(\vartheta) \Gamma(\vartheta)}\left\|f\left(\cdot, \varsigma_{n}(\cdot), \varsigma_{n}(\gamma(\cdot))\right)-f(\cdot, \varsigma(\cdot), \varsigma(\gamma(\cdot)))\right\| .
\end{aligned}
$$

Since $f$ is continuous, the operator $\mathbb{T}_{2}$ is also continuous. Thus, we have

$$
\left\|\left(\mathbb{T}_{2} \varsigma_{n}\right)-\left(\mathbb{T}_{2} \varsigma\right)\right\| \rightarrow 0 \quad \text { as } n \rightarrow \infty .
$$


Next, $\mathbb{T}_{2}$ is uniformly bounded on $B_{\xi}$. For any $\varsigma \in B_{\xi}$ and $\mathfrak{r} \in \mathfrak{Z}$, we have

$$
\begin{aligned}
\left|\left(\mathbb{T}_{2} \varsigma\right)\right| & =\max _{\mathfrak{r} \in \mathfrak{J}}\left|\left(\mathbb{T}_{2} \varsigma\right)(\mathfrak{r})\right| \\
& \leq \max _{\mathfrak{r} \in \mathfrak{J}}\left(\frac{1-\vartheta}{\mathfrak{N}(\vartheta)}|f(\mathfrak{r}, \varsigma(\mathfrak{r}), \varsigma(\gamma \mathfrak{r}))|+\frac{\vartheta}{\mathfrak{N}(\vartheta)} I_{a^{+}}^{\vartheta}|f(\mathfrak{r}, \varsigma(\mathfrak{r}), \varsigma(\gamma \mathfrak{r}))|\right) \\
& \leq \max _{\mathfrak{r} \in \mathfrak{J}}\left(\frac{(1-\vartheta)}{\mathfrak{N}(\vartheta)}\left(2 L_{f} \xi+\mu_{f}\right)+\frac{\left(2 L_{f} \xi+\mu_{f}\right)}{\mathfrak{N}(\vartheta)} \frac{(\mathfrak{r}-a)^{\vartheta}}{\Gamma(\vartheta)}\right) \\
& \leq \frac{2 L_{f} \xi+\mu_{f}}{\mathfrak{N}(\vartheta)}\left((1-\vartheta)+\frac{(T-a)^{\vartheta}}{\Gamma(\vartheta)}\right) .
\end{aligned}
$$

This leads to $\mathbb{T}_{2}$ is uniformly bounded on $B_{\xi}$.

Now, we show that $\mathbb{T}_{2}\left(B_{\xi}\right)$ is equicontinuous. For that, let $\varsigma \in B_{\xi}$ and $a \leq \mathfrak{r}_{1}<\mathfrak{r}_{2} \leq T$. Then, by using (4.8), we have

$$
\begin{aligned}
\left|\left(\mathbb{T}_{2} \varsigma\right)\left(\mathfrak{r}_{2}\right)-\left(\mathbb{T}_{2} \varsigma\right)\left(\mathfrak{r}_{1}\right)\right| \\
=\frac{1-\vartheta}{\mathfrak{N}(\vartheta)}\left|f\left(\mathfrak{r}_{2}, \varsigma\left(\mathfrak{r}_{2}\right), \varsigma\left(\gamma\left(\mathfrak{r}_{2}\right)\right)\right)-f\left(\mathfrak{r}_{1}, \varsigma\left(\mathfrak{r}_{1}\right), \varsigma\left(\gamma\left(\mathfrak{r}_{1}\right)\right)\right)\right| \\
\quad+\mid \frac{\vartheta}{\mathfrak{N}(\vartheta)} \frac{1}{\Gamma(\vartheta)} \int_{a}^{\mathfrak{r}_{2}}\left(\mathfrak{r}_{2}-\mathfrak{s}\right)^{\vartheta-1} f(\mathfrak{s}, \varsigma(\mathfrak{s}), \varsigma(\gamma(\mathfrak{s}))) d \mathfrak{s} \\
\quad-\frac{\vartheta}{\mathfrak{N}(\vartheta)} \frac{1}{\Gamma(\vartheta)} \int_{a}^{\mathfrak{r}_{1}}\left(\mathfrak{r}_{1}-\mathfrak{s}\right)^{\vartheta-1} f(\mathfrak{s}, \varsigma(\mathfrak{s}), \varsigma(\gamma(\mathfrak{s}))) d \mathfrak{s} \mid \\
\leq \frac{1-\vartheta}{\mathfrak{N}(\vartheta)}\left|f\left(\mathfrak{r}_{2}, \varsigma\left(\mathfrak{r}_{2}\right), \varsigma\left(\gamma\left(\mathfrak{r}_{2}\right)\right)\right)-f\left(\mathfrak{r}_{1}, \varsigma\left(\mathfrak{r}_{1}\right), \varsigma\left(\gamma\left(\mathfrak{r}_{1}\right)\right)\right)\right| \\
\quad+\frac{\vartheta}{\mathfrak{N}(\vartheta)} \frac{1}{\Gamma(\vartheta)} \int_{a}^{\mathfrak{r}_{1}}\left|\left(\mathfrak{r}_{1}-\mathfrak{s}\right)^{\vartheta-1}-\left(\mathfrak{r}_{2}-\mathfrak{s}\right)^{\vartheta-1}\right||f(\mathfrak{s}, \varsigma(\mathfrak{s}), \varsigma(\gamma(\mathfrak{s})))| d \mathfrak{s} \\
\quad+\frac{\vartheta}{\mathfrak{N}(\vartheta)} \frac{1}{\Gamma(\vartheta)} \int_{\mathfrak{r}_{1}}^{\mathfrak{r}_{2}}\left(\mathfrak{r}_{2}-\mathfrak{s}\right)^{\vartheta-1}|f(\mathfrak{s}, \varsigma(\mathfrak{s}), \varsigma(\gamma(\mathfrak{s})))| d \mathfrak{s} \\
\leq \frac{1-\vartheta}{\mathfrak{N}(\vartheta)}\left|f\left(\mathfrak{r}_{2}, \varsigma\left(\mathfrak{r}_{2}\right), \varsigma\left(\gamma\left(\mathfrak{r}_{2}\right)\right)\right)-f\left(\mathfrak{r}_{1}, \varsigma\left(\mathfrak{r}_{1}\right), \varsigma\left(\gamma\left(\mathfrak{r}_{1}\right)\right)\right)\right| \\
\quad+\frac{2\left(2 L_{f} \xi+\mu_{f}\right)}{\mathfrak{N}(\vartheta) \Gamma(\vartheta)}\left(\mathfrak{r}_{2}-\mathfrak{r}_{1}\right)^{\vartheta} .
\end{aligned}
$$

Since $f(\cdot, \varsigma(\cdot), \varsigma(\gamma(\cdot)))$ is continuous, $\left|\left(\mathbb{T}_{2} \varsigma\right)\left(\mathfrak{r}_{2}\right)-\left(\mathbb{T}_{2} \varsigma\right)\left(\mathfrak{r}_{1}\right)\right| \rightarrow 0$ as $\mathfrak{r}_{2} \rightarrow \mathfrak{r}_{1}$. In light of the former steps with Arzela-Ascoli theorem, we derive that $\left(\mathbb{T}_{2} B_{\xi}\right)$ is relatively compact, and hence $\mathbb{T}_{2}$ is completely continuous. So, Theorem 2.2 shows that (1.1) has at least one solution.

\section{Ulam-Hyers stability}

In this section, we discuss two types of stability for (1.1), namely Ulam-Hyers and generalized Ulam-Hyers stabilities. For $\varepsilon>0$, we consider the following inequations:

$$
\left|A B C \mathbb{D}_{a^{+}}^{\vartheta} \widetilde{\zeta}(\mathfrak{r})-f(\mathfrak{r}, \widetilde{\zeta}(\mathfrak{r}), \widetilde{\zeta}(\gamma \mathfrak{r}))\right| \leq \varepsilon, \quad \mathfrak{r} \in \mathfrak{Z}
$$

Definition 5.1 The pantograph ABC-FDEs (1.1) are Ulam-Hyers stable if there exists a real number $C_{f}>0$ such that, for each $\varepsilon>0$ and for each solution $\widetilde{\varsigma} \in \mathfrak{D}$ of inequality (5.1), 
there exists a unique solution $\varsigma \in \mathfrak{D}$ of (1.1) with

$$
|\widetilde{\zeta}(\mathfrak{r})-\varsigma(\mathfrak{r})| \leq C_{f} \varepsilon, \quad \mathfrak{r} \in \mathfrak{Z} .
$$

And the pantograph ABC-FDEs (1.1) are generalized Ulam-Hyers stable if we can find $\phi_{f}: \mathbb{R}^{+} \rightarrow \mathbb{R}^{+}$with $\phi_{f}(0)=0$ such that

$$
|\widetilde{\zeta}(\mathfrak{r})-\varsigma(\mathfrak{r})| \leq \phi_{f}(\varepsilon), \quad \mathfrak{r} \in \mathfrak{Z}
$$

Remark 5.1 Let $\widetilde{\varsigma} \in \mathfrak{D}$ be the solution of inequality (5.1) if and only if we have a function $h \in \mathfrak{D}$ which depends on $\widetilde{\varsigma}$ such that

i) $|h(\mathfrak{r})| \leq \varepsilon$ for all $\mathfrak{r} \in \mathfrak{Z}$,

ii) $A B C \mathbb{D}_{a^{+}}^{\vartheta} \widetilde{\zeta}(\mathfrak{r})=f(\mathfrak{r}, \widetilde{\zeta}(\mathfrak{r}), \widetilde{\varsigma}(\gamma \mathfrak{r}))+h(\mathfrak{r}), \mathfrak{r} \in \mathfrak{Z}$.

Lemma 5.1 If $\widetilde{\varsigma} \in \mathfrak{D}$ is a solution of inequality (5.1), then $\widetilde{\varsigma}$ is a solution of the following inequality:

$$
\left|\widetilde{\zeta}(\mathfrak{r})-\mathcal{R}_{\widetilde{\zeta}}-{ }^{A B} \mathbb{I}_{a^{+}}^{\vartheta} f(\mathfrak{r}, \widetilde{\zeta}(\mathfrak{r}), \widetilde{\zeta}(\gamma \mathfrak{r}))\right| \leq \Lambda \varepsilon
$$

where

$$
\mathcal{R}_{\widetilde{\zeta}}:=\frac{1}{1-\sum_{k=1}^{m} c_{k}} \sum_{k=1}^{m} c_{k}^{A B} \mathbb{I}_{a^{+}}^{\vartheta} f\left(\mathfrak{r}_{k}, \widetilde{\zeta}\left(\mathfrak{r}_{k}\right), \widetilde{\zeta}\left(\gamma \mathfrak{r}_{k}\right)\right)
$$

and

$$
\Lambda:=\left(\frac{1-\vartheta}{1-\sum_{k=1}^{m} c_{k}}+\frac{1}{\Gamma(\vartheta)}\left(\frac{1}{1-\sum_{k=1}^{m} c_{k}} \sum_{k=1}^{m} c_{k}\left(\mathfrak{r}_{k}-a\right)\right)^{\vartheta}+(T-a)\right)^{\vartheta} \frac{1}{\mathfrak{N}(\vartheta)} .
$$

Proof In view of Remark 5.1, we have

$$
\begin{aligned}
& { }^{A B C} \mathbb{D}_{a^{+}}^{\vartheta} \widetilde{\zeta}(\mathfrak{r})=f(\mathfrak{r}, \widetilde{\zeta}(\mathfrak{r}), \widetilde{\zeta}(\gamma \mathfrak{r}))+h(\mathfrak{r}), \quad \mathfrak{r} \in \mathfrak{Z}, \\
& \widetilde{\zeta}(a)=\sum_{k=1}^{m} c_{k} \widetilde{\zeta}\left(\mathfrak{r}_{k}\right), \quad \mathfrak{r}_{k} \in \mathfrak{Z}^{\prime} .
\end{aligned}
$$

Then, by Theorem 3.1, we get

$$
\begin{aligned}
\widetilde{\zeta}(\mathfrak{r})= & \frac{1}{1-\sum_{k=1}^{m} c_{k}} \sum_{k=1}^{m} c_{k}^{A B} \mathbb{I}_{a^{+}}^{\vartheta}\left(f\left(\mathfrak{r}_{k}, \widetilde{\zeta}\left(\mathfrak{r}_{k}\right), \widetilde{\zeta}\left(\gamma \mathfrak{r}_{k}\right)\right)+h\left(\mathfrak{r}_{k}\right)\right) \\
& +{ }^{A B} \mathbb{I}_{a^{+}}^{\vartheta}(f(\mathfrak{r}, \widetilde{\zeta}(\mathfrak{r}), \widetilde{\zeta}(\gamma \mathfrak{r}))+h(\mathfrak{r})) \\
= & \mathcal{R}_{\widetilde{\zeta}}+\frac{1}{1-\sum_{k=1}^{m} c_{k}} \sum_{k=1}^{m} c_{k}{ }^{A B} \mathbb{I}_{a^{+}}^{\vartheta} h\left(\mathfrak{r}_{k}\right)+{ }^{A B} \mathbb{I}_{a^{+}}^{\vartheta}(f(\mathfrak{r}, \widetilde{\zeta}(\mathfrak{r}), \widetilde{\zeta}(\gamma \mathfrak{r}))+h(\mathfrak{r})) .
\end{aligned}
$$


From this it follows that

$$
\begin{aligned}
&\left|\widetilde{\zeta}(\mathfrak{r})-\mathcal{R}_{\widetilde{\zeta}}-{ }^{A B} \mathbb{I}_{a^{+}}^{\vartheta} f(\mathfrak{r}, \widetilde{\zeta}(\mathfrak{r}), \widetilde{\zeta}(\gamma \mathfrak{r}))\right| \\
& \leq \frac{1}{1-\sum_{k=1}^{m} c_{k}} \sum_{k=1}^{m} c_{k}{ }^{A B} \mathbb{I}_{a^{+}}^{\vartheta}\left|h\left(\mathfrak{r}_{k}\right)\right|+{ }^{A B} \mathbb{I}_{a^{+}}^{\vartheta}|h(\mathfrak{r})| \\
&= \frac{1}{1-\sum_{k=1}^{m} c_{k}} \sum_{k=1}^{m} c_{k}\left(\frac{1-\vartheta}{\mathfrak{N}(\vartheta)}\left|h\left(\mathfrak{r}_{k}\right)\right|+\frac{\vartheta}{\mathfrak{N}(\vartheta)} I_{a^{+}}^{\vartheta}\left|h\left(\mathfrak{r}_{k}\right)\right|\right) \\
&+\frac{1-\vartheta}{\mathfrak{N}(\vartheta)}|h(\mathfrak{r})|+\frac{\vartheta}{\mathfrak{N}(\vartheta)} I_{a^{+}}^{\vartheta}|h(\mathfrak{r})| \\
& \leq\left(\frac{1-\vartheta}{1-\sum_{k=1}^{m} c_{k}}+\frac{1}{\Gamma(\vartheta)}\left(\frac{1}{1-\sum_{k=1}^{m} c_{k}} \sum_{k=1}^{m} c_{k}\left(\mathfrak{r}_{k}-a\right)\right)^{\vartheta}+(T-a)\right) \frac{\varepsilon}{\mathfrak{N}(\vartheta)} \\
&= \Lambda \varepsilon .
\end{aligned}
$$

Theorem 5.1 Suppose that hypothesis $\left(A_{1}\right)$ holds. If $\mathfrak{N}(\vartheta)-(1-\vartheta) 2 L_{f}<1$, then the pantograph ABC-FDEs (1.1) are Ulam-Hyers stable.

Proof Let $\varepsilon>0$ and $\widetilde{\zeta} \in \mathfrak{D}$ be a function which satisfies inequality (5.1), and let $\varsigma \in \mathfrak{D}$ be the unique solution of the following problem:

$$
\begin{cases}A B C \mathbb{D}_{a^{+}}^{\vartheta} \zeta(\mathfrak{r})=f(\mathfrak{r}, \varsigma(\mathfrak{r}), \varsigma(\gamma \mathfrak{r})), & \mathfrak{r} \in \mathfrak{Z}, \\ \varsigma(a)=\sum_{k=1}^{m} c_{k} \zeta\left(\mathfrak{r}_{k}\right)=\sum_{k=1}^{m} c_{k} \widetilde{\zeta}\left(\mathfrak{r}_{k}\right)=\widetilde{\varsigma}(a), & \mathfrak{r}_{k} \in \mathfrak{Z}^{\prime}\end{cases}
$$

Using Theorem 3.1, we obtain

$$
\zeta(\mathfrak{r})=\mathcal{R}_{\varsigma}+{ }^{A B} \mathbb{I}_{a^{+}}^{\vartheta} f(\mathfrak{r}, \varsigma(\mathfrak{r}), \varsigma(\gamma \mathfrak{r}))
$$

where

$$
\mathcal{R}_{\varsigma}:=\frac{1}{1-\sum_{k=1}^{m} c_{k}} \sum_{k=1}^{m} c_{k}^{A B} \mathbb{I}_{a^{+}}^{\vartheta} f\left(\mathfrak{r}_{k}, \varsigma\left(\mathfrak{r}_{k}\right), \varsigma\left(\gamma \mathfrak{r}_{k}\right)\right)
$$

Since $\zeta(a)=\widetilde{\zeta}(a)$ and $\sum_{k=1}^{m} c_{k} \varsigma\left(\mathfrak{r}_{k}\right)=\sum_{k=1}^{m} c_{k} \widetilde{\zeta}\left(\mathfrak{r}_{k}\right)$, then $\mathcal{R}_{\varsigma}=\mathcal{R}_{\widetilde{\zeta}}$. Hence

$$
\varsigma(\mathfrak{r})=\mathcal{R}_{\widetilde{\varsigma}}+{ }^{A B} \mathbb{I}_{a^{+}}^{\vartheta} f(\mathfrak{r}, \varsigma(\mathfrak{r}), \varsigma(\gamma \mathfrak{r})) .
$$

It follows from Lemma 5.1 and $\left(A_{1}\right)$ that

$$
\begin{aligned}
\mid \widetilde{\zeta}(\mathfrak{r}) & -\zeta(\mathfrak{r}) \mid \\
\leq & \left|\widetilde{\zeta}(\mathfrak{r})-\mathcal{R}_{\widetilde{\zeta}}-{ }^{A B} \mathbb{I}_{a^{+}} f(\mathfrak{r}, \widetilde{\zeta}(\mathfrak{r}), \widetilde{\zeta}(\gamma \mathfrak{r}))\right| \\
& +\left|{ }^{A B} \mathbb{I}_{a^{+}}^{\vartheta} f(\mathfrak{r}, \widetilde{\zeta}(\mathfrak{r}), \widetilde{\zeta}(\gamma \mathfrak{r}))-{ }^{A B} \mathbb{I}_{a^{+}}^{\vartheta} f(\mathfrak{r}, \zeta(\mathfrak{r}), \zeta(\gamma \mathfrak{r}))\right| \\
\leq & \Lambda \varepsilon+{ }^{A B} \mathbb{I}_{a^{+}}^{\vartheta}|f(\mathfrak{r}, \widetilde{\zeta}(\mathfrak{r}), \widetilde{\zeta}(\gamma \mathfrak{r}))-f(\mathfrak{r}, \zeta(\mathfrak{r}), \zeta(\gamma \mathfrak{r}))| \\
\leq & \Lambda \varepsilon+2 L_{f}{ }^{A B} \mathbb{I}_{a^{+}}^{\vartheta}|\widetilde{\zeta}(\mathfrak{r})-\varsigma(\mathfrak{r})| .
\end{aligned}
$$


Using Theorem 2.3 with $\sigma(\mathfrak{r})=|\widetilde{\zeta}(t)-\varsigma(t)|, a(\mathfrak{r})=\Lambda \varepsilon$, and $b(\mathfrak{r})=2 L_{f}$, we get

$$
\begin{aligned}
|\widetilde{\zeta}(\mathfrak{r})-\varsigma(\mathfrak{r})| & \leq \frac{\Lambda \varepsilon \mathfrak{N}(\vartheta)}{\mathfrak{N}(\vartheta)-(1-\vartheta) 2 L_{f}} \mathbb{E}_{\vartheta}\left(\frac{2 \vartheta L_{f}(\mathfrak{r}-a)^{\vartheta}}{\mathfrak{N}(\vartheta)-2(1-\vartheta) L_{f}}\right) \\
& \leq C_{f} \varepsilon,
\end{aligned}
$$

where

$$
C_{f}:=\frac{\Lambda \mathfrak{N}(\vartheta)}{\mathfrak{N}(\vartheta)-(1-\vartheta) 2 L_{f}} \mathbb{E}_{\vartheta}\left(\frac{2 \vartheta L_{f}(T-a)^{\vartheta}}{\mathfrak{N}(\vartheta)-2(1-\vartheta) L_{f}}\right)
$$

Corollary 5.1 Under the hypotheses of Theorem 5.1, if there exists $\phi_{f}: \mathbb{R}^{+} \rightarrow \mathbb{R}^{+}$with $\phi_{f}(0)=0$, then the pantograph $A B C$ problem (1.1) has generalized Ulam-Hyers stability.

Proof Choosing $\phi_{f}(\varepsilon)=C_{f} \varepsilon$ and $\phi_{f}(0)=0$, from Theorem 5.1 we obtain $|\widetilde{\zeta}(\mathfrak{r})-\varsigma(\mathfrak{r})| \leq$ $\phi_{f}(\varepsilon)$.

\section{Examples}

In this section, we justify the validity of Theorems 4.1, 4.2, and 5.1 through an example.

Example 6.1 For $\vartheta \in(0,1]$, we consider the following $\mathrm{ABC}$ fractional problem:

$$
\left\{\begin{array}{l}
A B C \mathbb{D}_{0^{+}}^{\frac{1}{2}} \zeta(\mathfrak{r})=\frac{\mathfrak{r}^{2}}{10}\left(e^{-\mathfrak{r}}+\frac{|\zeta(\mathfrak{r})|}{10(1+|\zeta(\mathfrak{r})|)}+\frac{\left|\zeta\left(\frac{\mathfrak{r}}{2}\right)\right|}{10\left(1+\left|\zeta\left(\frac{\mathfrak{r}}{2}\right)\right|\right)}\right), \quad \mathfrak{r} \in[0,1], \\
\varsigma(0)=\frac{1}{4} \zeta\left(\frac{1}{3}\right)
\end{array}\right.
$$

where $\vartheta=\frac{1}{2}, c_{1}=\frac{1}{4}, \mathfrak{r}_{1}=\frac{1}{3}(m=1), f(\mathfrak{r}, \varsigma(\mathfrak{r}), \varsigma(\gamma \mathfrak{r}))=\frac{\mathfrak{r}^{2}}{10}\left(e^{-\mathfrak{r}}+\frac{|\zeta(\mathfrak{r})|}{10(1+|\zeta(\mathfrak{r})|)}+\frac{\left|\zeta\left(\frac{\mathfrak{r}}{2}\right)\right|}{10\left(1+\left|\zeta\left(\frac{\mathfrak{r}}{2}\right)\right|\right)}\right)$, and $\gamma=\frac{1}{2}$.

Clearly, $f(0, \varsigma(0), \bar{\zeta}(0))=0$. Moreover,

$$
\mu_{f}=\max _{\mathfrak{r} \in[0,1]}|f(\mathfrak{r}, 0,0)|=\max _{\mathfrak{r} \in[0,1]} \frac{\mathfrak{r}^{2}}{10} e^{-\mathfrak{r}}=\frac{1}{10 e} .
$$

Let $\mathfrak{r} \in[0,1]$ and $\varsigma, v \in \mathbb{R}$. Then

$$
\begin{aligned}
\mid f\left(\mathfrak{r}, \varsigma(\mathfrak{r}), \varsigma\left(\frac{1}{2} \mathfrak{r}\right)-f\left(\mathfrak{r}, v(\mathfrak{r}), v\left(\frac{1}{2} \mathfrak{r}\right) \mid\right.\right. \\
\leq \mid \frac{\mathfrak{r}^{2}}{10}\left(e^{-\mathfrak{r}}+\frac{|\zeta(\mathfrak{r})|}{10(1+|\zeta(\mathfrak{r})|)}+\frac{\left|\zeta\left(\frac{\mathfrak{r}}{2}\right)\right|}{10\left(1+\left|\zeta\left(\frac{\mathfrak{r}}{2}\right)\right|\right)}\right) \\
\quad-\frac{\mathfrak{r}^{2}}{10}\left(e^{-\mathfrak{r}}+\frac{|v(\mathfrak{r})|}{10(1+|v(\mathfrak{r})|)}+\frac{\left|v\left(\frac{\mathfrak{r}}{2}\right)\right|}{10\left(1+\left|v\left(\frac{\mathfrak{v}}{2}\right)\right|\right)}\right) \mid \\
\leq \frac{1}{10}\left(\frac{10|\zeta(\mathfrak{r})-v(\mathfrak{r})|}{100(1+|\zeta(\mathfrak{r})|)(1+|v(\mathfrak{r})|)}+\frac{10\left|\zeta\left(\frac{\mathfrak{r}}{2}\right)-v\left(\frac{\mathfrak{r}}{2}\right)\right|}{100\left(1+\left|\zeta\left(\frac{\mathfrak{r}}{2}\right)\right|\right)\left(1+\left|v\left(\frac{\mathfrak{r}}{2}\right)\right|\right)}\right) \\
\leq \frac{1}{10}\left(|\zeta(\mathfrak{r})-v(\mathfrak{r})|+\left|\zeta\left(\frac{\mathfrak{r}}{2}\right)-v\left(\frac{\mathfrak{r}}{2}\right)\right|\right) .
\end{aligned}
$$


Therefore, hypothesis $\left(A_{1}\right)$ holds with $L_{f}=\frac{1}{10}$. We shall examine that condition (4.1) is satisfied with $\mathfrak{N}(\vartheta)=1$ and $m=1$. Hence, by some simple calculations, we find that

$$
\mathfrak{P}_{1}=\left(\frac{1}{15}+\frac{9+\sqrt{3}}{45 \sqrt{\pi}}\right) \approx 0.2<1
$$

Thus Theorems 4.1 and 4.2 guarantee the existence and uniqueness of solution on $[0,1]$ problem (6.1).

Finally, since $\mathfrak{N}(\vartheta)-(1-\vartheta) 2 L_{f}=\frac{9}{10}<1$, problem (6.1) is Ulam-Hyers and generalized Ulam-Hyers stable with

$$
C_{f}=\frac{10}{9} \Lambda \mathbb{E}_{\frac{1}{2}}\left(\frac{1}{9}\right) \text { and } \Lambda=\left(\frac{1}{3}+\frac{9+\sqrt{3}}{9 \sqrt{\pi}}\right) .
$$

\section{Remark 6.1}

(1) If $\gamma=1$, then our problem (1.1) reduces to problem (1.2). Therefore, all the results mentioned in this work are also valid for problem (1.2).

(2) If we replace the nonlocal condition $\varsigma(a)=\sum_{k=1}^{m} c_{k} \varsigma\left(\mathfrak{r}_{k}\right)$ with the initial condition $\varsigma(0)=\varsigma_{0}(a=0)$ and use ${ }^{C F} \mathbb{D}_{a^{+}}^{\vartheta}$ (Caputo-Fabrizio FD) instead of ${ }^{A B C} \mathbb{D}_{a^{+}}^{\vartheta}(\mathrm{ABC}$ derivative), then our problem (1.1) reduces to the following problem:

$$
\left\{\begin{array}{l}
C F \mathbb{D}_{0^{+}}^{\vartheta} \varsigma(\mathfrak{r})=f(\mathfrak{r}, \varsigma(\mathfrak{r}), \varsigma(\gamma \mathfrak{r})), \quad \mathfrak{r} \in[a, T], 0<\vartheta \leq 1, \\
\varsigma(0)=\varsigma_{0} .
\end{array}\right.
$$

\section{Conclusions}

The theory of fractional operators including nonsingular kernels is novel and of significant recent interest, thus there is a need to study the qualitative properties of FDEs involving such operators. In this paper, we have obtained the existence and uniqueness of solutions for the pantograph FDEs with nonlocal conditions involving ABC fractional derivative. Our approach is based on the reduction of ABC-type pantograph FDEs into FIE and some fixed point theorems of Banach and Krasnoselskii. Further, we have applied Gronwall's inequality in the frame of $\mathrm{AB}$ fractional integral operator to develop adequate results for various types of Ulam-Hyers stability. Pertinent examples are provided to justify the results. The problems scrutinized are also valid for some special cases, in other words, they are reduced to the corresponding problems that contain Caputo-Fabrizio fractional derivative operator. Besides, the analysis of the obtained results was restricted to a minimum of assumptions.

\section{Acknowledgements}

All authors have read and approved the last draft of the paper.

\section{Funding}

This research was funded by the Deanship of Scientific Research at Princess Nourah bint Abdulrahman University through the Fast-track Research Funding Program.

Availability of data and materials

Not applicable. 
Authors' contributions

All authors contributed equally to this work. All authors read and approved the final manuscript.

\section{Author details}

${ }^{1}$ Department of Mathematics, Hodeidah University, Al-Hodeidah, Yemen. ${ }^{2}$ Department of Mathematics and General Sciences, Prince Sultan University, Riyadh, Saudi Arabia. ${ }^{3}$ Department of Medical Research, China Medical University, Taichung 40402, Taiwan. ${ }^{\circ}$ Department of Computer Science and Information Engineering, Asia University, Taichung, Taiwan. ${ }^{5}$ Department of Mathematics, Shivaji University, Kolhapur 416004, Maharashtra, India. ${ }^{6}$ Department of Mathematical Sciences, Princess Nourah bint Abdulrahman University, Riyadh, Saudi Arabia. ${ }^{7}$ Department of Basic Engineering Sciences, College of Engineering, Imam Abdulrahman Bin Faisal University, P.O. Box 1982, Dammam, 34151 , Saudi Arabia. ${ }^{8}$ Department of Mathematics, Imam Muhammed Ibn Saud Islamic University, Riyadh, Saudi Arabia.

\section{Publisher's Note}

Springer Nature remains neutral with regard to jurisdictional claims in published maps and institutional affiliations.

Received: 9 November 2020 Accepted: 11 January 2021 Published online: 21 January 2021

\section{References}

1. Podlubny, I.: Fractional Differential Equations. Academic Press, San Diego (1999)

2. Samko, S.G., Kilbas, A.A., Marichev, O.I., et al.: Fractional Integrals and Derivatives. Gordon \& Breach, Yverdon (1993)

3. Diethelm, K.: The analysis of fractional differential equations. 2004 of lecture notes in mathematics, (2010)

4. Magin, R.L.: Fractional Calculus in Bioengineering. 2. Begell House, Redding (2006)

5. Kilbas, A.A., Srivastava, H.M., Trujillo, J.J.: Theory and Applications of Fractional Differential Equations. North-Holland Mathematics Studies. Elsevier, Amsterdam (2006)

6. Machado, J.T., Kiryakova, V., Mainardi, F.: Recent history of fractional calculus. Commun. Nonlinear Sci. Numer. Simul. 16(3), 1140-1153 (2011)

7. Hilfer, R., et al.: Applications of Fractional Calculus in Physics, vol. 35. World Scientific, Singapore (2000)

8. Lorenzo, C.F., Hartley, T.T.: Variable order and distributed order fractional operators. Nonlinear Dyn. 29(1-4), 57-98 (2002)

9. Agrawal, O.P.: Formulation of Euler-Lagrange equations for fractional variational problems. J. Math. Anal. Appl. 272(1), 368-379 (2002)

10. Losada, J., Nieto, J.J.: Properties of a new fractional derivative without singular kernel. Prog. Fract. Differ. Appl. 1(2), 87-92 (2015)

11. Caputo, M., Fabrizio, M.: A new definition of fractional derivative without singular kernel. Prog. Fract. Differ. Appl. 1(2), $1-13(2015)$

12. Khalil, R., Al Horani, M., Yousef, A., Sababheh, M.: A new definition of fractional derivative. J. Comput. Appl. Math. 264, 65-70 (2014)

13. Jarad, F., Abdeljawad, T., Hammouch, Z.: On a class of ordinary differential equations in the frame of Atangana-Baleanu fractional derivative. Chaos Solitons Fractals 117, 16-20 (2018)

14. Atangana, A., Baleanu, D.: New fractional derivatives with nonlocal and nonsingular kernel: theory and application to heat transfer model (2016). arXiv:1602.03408. arXiv preprint

15. Atangana, A., Koca, I.: Chaos in a simple nonlinear system with Atangana-Baleanu derivatives with fractional order Chaos Solitons Fractals 89, 447-454 (2016)

16. Alkahtani, B.S.T.: Chua's circuit model with Atangana-Baleanu derivative with fractional order. Chaos Solitons Fractals 89, 547-551 (2016)

17. Abdeljawad, T., Baleanu, D.: Discrete fractional differences with nonsingular discrete Mittag-Leffler kernels. Adv. Differ Equ. 2016(1), 232 (2016)

18. Abdeljawad, T., Baleanu, D.: On fractional derivatives with exponential kernel and their discrete versions. Rep. Math. Phys. 80(1), 11-27 (2017)

19. Singh, J., Kumar, D., Hammouch, Z., Atangana, A.: A fractional epidemiological model for computer viruses pertaining to a new fractional derivative. Appl. Math. Comput. 316, 504-515 (2018)

20. Uïcar, S., Uïcar, E., Ozdemir, N., Hammouch, Z:: Mathematical analysis and numerical simulation for a smoking model with Atangana-Baleanu derivative. Chaos Solitons Fractals 118, 300-306 (2019)

21. Atangana, A.: Modelling the spread of Covid-19 with new fractal-fractional operators: can the lockdown save mankind before vaccination. Chaos Solitons Fractals 136, 109860 (2020)

22. Zhang, Z:: A novel COVID-19 mathematical model with fractional derivatives: singular and nonsingular kernels. Chaos Solitons Fractals 139, 110060 (2020)

23. Behzad, G., Atangana, A.: A new application of fractional Atangana-Baleanu derivatives: designing ABC-fractional masks in image processing. Phys. A, Stat. Mech. Appl. 542, 123516 (2020)

24. Atangana, A., Gómez-Aguilar, J.F.: Fractional derivatives with no-index law property: application to chaos and statistics. Chaos Solitons Fractals 114, 516-535 (2018)

25. Atangana, A.: Fractal-fractional differentiation and integration: connecting fractal calculus and fractional calculus to predict complex system. Chaos Solitons Fractals 102, 396-406 (2017)

26. Toufik, M., Atangana, A.: New numerical approximation of fractional derivative with non-local and non-singular kernel: application to chaotic models. Eur. Phys. J. Plus 132, 444 (2017). https://doi.org/10.1140/epjp/i2017-11717-0

27. Abdo, M.S., Panchal, S.K.: Fractional integro-differential equations involving $\psi$-Hilfer fractional derivative. Adv. Appl. Math. Mech. 11(1), 1-22 (2019)

28. Abdo, M.S., Panchal, S.K., Saeed, A.M.: Fractional boundary value problem with $\psi$-Caputo fractional derivative. Proc. Math. Sci. 129(5), 65 (2019)

29. Ahmad, B., Ntouyas, S.K., Alsaedi, A.: Sequential fractional differential equations and inclusions with semi-periodic and nonlocal integro-multipoint boundary conditions. J. King Saud Univ., Sci. 31(2), 184-193 (2019) 
30. Gu, H., Trujillo, J.J.: Existence of mild solution for evolution equation with Hilfer fractional derivative. Appl. Math. Comput. 257, 344-354 (2015)

31. Abdeljawad, T:: A Lyapunov type inequality for fractional operators with nonsingular Mittag-Leffler kernel. J. Inequal. Appl. 2017(1), 130 (2017)

32. Abdo, M.S., Shah, K., Wahash, H.A., Panchal, S.K.: On a comprehensive model of the novel coronavirus (Covid-19) under Mittag-Leffler derivative. Chaos Solitons Fractals 2020, 109867 (2020). https://doi.org/10.1016/j.chaos.2020.109867

33. Abdo, M.S., Abdeljawad, T., Shah, K., Jarad, F.: Study of impulsive problems under Mittag-Leffler power law. Heliyon 6(10), e05109 (2020)

34. Redhwan, S.S., Abdo, M.S., Shah, K., Abdeljawad, T., Dawood, S., Abdo, H.A., Shaikhh, S.L.: Mathematical modeling for the outbreak of the coronavirus (COVID-19) under fractional nonlocal operator. Results Phys. 19, 103610 (2020). https://doi.org/10.1016/j.rinp.2020.103610

35. Thabet, S.T.M., Abdo, M.S., Shah, K., Abdeljawad, T.: Study of transmission dynamics of COVID-19 mathematical model under ABC fractional order derivative. Results Phys. 19, 103507 (2020). https://doi.org/10.1016/j.rinp.2020.103507

36. Yavuz, M., Ozdemir, N., Baskonus, H.M.: Solutions of partial differential equations using the fractional operator involving Mittag-Leffler kernel. Eur. Phys. J. Plus 133(6), 215 (2018)

37. Kucche, K.D., Sutar, S.T.: Analysis of nonlinear fractional differential equations involving Atangana-Baleanu-Caputo derivative. Chaos Solitons Fractals 143, 110556 (2020)

38. Sutar, S.T., Kucche, K.D.: On nonlinear hybrid fractional differential equations with Atangana-Baleanu-Caputo derivative (2020). arXiv:2007.11034. arXiv preprint

39. Ockendon, J.R., Tayler, A.B.: The dynamics of a current collection system for an electric locomotive. Proc. R. Soc. Lond. Ser. A, Math. Phys. Sci. 322(1551), 447-468 (1971)

40. Balachandran, K., Kiruthika, S., Trujillo, J.J.: Existence of solutions of nonlinear fractional pantograph equations. Acta Math. Sci. 33(3), 712-720 (2013)

41. Vivek, D., Kanagarajan, K., Sivasundaram, S.: Theory and analysis of nonlinear neutral pantograph equations via Hilfer fractional derivative. Nonlinear Stud. 24(3), 699-712 (2017)

42. Anguraj, A., Vinodkumar, A., Malar, K.: Existence and stability results for random impulsive fractional pantograph equations. Filomat 30(14), 3839-3854 (2016)

43. Vivek, D., Kanagarajan, K., Harikrishnan, S.: Dynamics and stability of Hilfer-Hadamard type fractional pantograph equations with boundary conditions. J. Nonlinear Anal. Appl. 2018(1), 1-13 (2018)

44. Elsayed, E., Harikrishnan, S., Kanagarajan, K.: Analysis of nonlinear neutral pantograph differential equations with Hilfer fractional derivative. MathLAB 1, 231-240 (2018)

45. Harikrishnan, S., Ibrahim, R., Kanagarajan, K.: Establishing the existence of Hilfer fractional pantograph equations with impulses. Fundam. J. Math. Appl. 1(1), 36-42 (2018)

46. Ahmed, I., Kumam, P., Shah, K., et al.: Stability results for implicit fractional pantograph differential equations via Hilfer fractional derivative with a nonlocal Riemann-Liouville fractional integral condition. Mathematics 8(1), 94 (2020)

47. Borisut, P., Kumam, P., Ahmed, I., Sitthithakerngkiet, K.: Nonlinear Caputo fractional derivative with nonlocal Riemann-Liouville fractional integral condition via fixed point theorems. Symmetry 11(6), 829 (2019)

48. Ahmed, I., Kumam, P., Shah, K., Borisut, P., Sitthithakerngkiet, K., Ahmed, D.: Stability results for implicit fractional pantograph differential equations via $\phi$-Hilfer fractional derivative with a nonlocal Riemann-Liouville fractional integral condition. Mathematics 8(1), 94 (2020)

49. Borisut, P., Kumam, P., Ahmed, I., Jirakitpuwapat, W.: Existence and uniqueness for $\psi$-Hilfer fractional differential equation with nonlocal multi-point condition. Math. Methods Appl. Sci. (2020). https://doi.org/10.1002/mma.6092

50. Ahmed, I., Kumam, P., Jarad, F., Borisut, P., Sitthithakerngkiet, K., Ibrahim, A.: Stability analysis for boundary value problems with generalized nonlocal condition via Hilfer-Katugampola fractional derivative. Adv. Differ. Equ. 2020(1) 225 (2020)

51. Borisut, P., Kumam, P., Ahmed, l., Sitthithakerngkiet, K.: Positive solution for nonlinear fractional differential equation with nonlocal multi-point condition. Fixed Point Theory 21(2), 427-440 (2020)

52. Zhou, Y., Wang, J., Zhang, L.: Basic Theory of Fractional Differential Equations. World Scientific, Singapore (2016)

\section{Submit your manuscript to a SpringerOpen ${ }^{\circ}$ journal and benefit from:}

- Convenient online submission

- Rigorous peer review

- Open access: articles freely available online

- High visibility within the field

- Retaining the copyright to your article

Submit your next manuscript at $\gg$ springeropen.com 